\title{
A CONSTRUCTIVE APPROACH TO THE THEORY OF STOCHASTIC PROCESSES $\left({ }^{1}\right)$
}

\author{
BY \\ YUEN-KWOK CHAN
}

\begin{abstract}
Some basic problems in probability theory will be considered with the constructive point of view. Among them are the construction of measurable stochastic processes from finite joint probabilities, and the construction of interesting random variables related to a given process. These random variables include (1) the first instant when a process has spent a definite length of time in a definite set, and (2) the value of another process at such an instant.
\end{abstract}

1. Introduction and preliminaries. This paper is hoped to contribute to the constructive program of developing mathematics which have computational content and in which every assertion of existence is, explicitly or implicitly, accompanied by a finite routine of construction. The integration and measure theory developed by Bishop in [1] and by Bishop and Cheng in [2] will be used freely. The difference between an argument used and its classical counterpart will be pointed out if the difference is basic.

Basically a probability space is defined as a triple $(\Omega, L, E)$ where $\Omega$ is a set with an equality relation and an inequality relation, $L$ is a family of functions defined on subsets of $\Omega$, and $E$ is a function on $L$, satisfying the following four conditions.

(1) $L$ is closed under addition and scalar multiplication, and contains the functions which are constant on the whole of $\Omega$.

(2) $E$ is linear on $L$ with $E(1)=1$.

(3) If $X, Y$ are in $L$, then so is $X \wedge Y(=\min (X, Y))$, and $\lim _{n \rightarrow \infty} E(X \wedge n)$ $=E(X)$.

(4) If $X_{0}, X_{1}, X_{2}, \ldots$ is a sequence of nonnegative functions in $L$ such that $\sum_{n=1}^{\infty} E\left(X_{n}\right)$ converges and is less than $E\left(X_{0}\right)$, then there exists a point $\omega$ which is in the domain of $X_{n}$ for every $n$ and such that $\sum_{n=1}^{\infty} X_{n}(\omega)$ converges and is less than $X_{0}(\omega)$.

It should be emphasized that by a function on $\Omega$ we mean one defined on a subset of $\Omega$. Condition (4) implies, among other things, that the functions belonging to $L$ will have nonempty domains. The function $E$ induces a norm on $L$ by $\|X\|$ $=E(|X|)$. The function space $L$ can be enlarged to $L^{1}=L^{1}(E)$, and $E$ extended

Received by the editors November 3, 1970.

AMS 1969 subject classifications. Primary 6002, 6040; Secondary 0203.

Key words and phrases. Constructive mathematics, probability spaces, measurable stochastic processes, Kolmogorov's Theorem, first crossing times, stopping times.

(1) This research was supported in part by NSF Grant 20047.

Copyright (C) 1972, American Mathematical Society 
accordingly, so that $\left(\Omega, L^{1}, E\right)$ is a probability space where $L^{1}$ is complete with respect to this norm. In the following we also write $\int X(\omega) d E(\omega)$ for $E(X)$. The triple $\Omega, L^{1}, E$ thus represents the sample space, the family of integrable functions (or integrable random variables), and the expectation function. (This approach is found to be more natural than the usual definition where a probability space consists of a sample space, the measurable sets, and a probability function.) An important example: if $(\Omega, d)$ is a locally compact metric space and if $E$ is a nonnegative linear functional on $C(\Omega)$, the space of continuous functions with compact supports, such that for some sequence $X_{n}$ in $C(\Omega)$ which converges to 1 uniformly on compact subsets of $\Omega$ the sequence $E\left(X_{n}\right)$ converges to 1 , then $E$ can be extended to $B C(\Omega)$, the bounded continuous functions, and $(\Omega, B C(\Omega), E)$ is a probability space. In this case we will (abuse the terminology and) say $(\Omega, C(\Omega), E)$ is a probability space. Suppose $(\Omega, L, E)$ is a given probability space. If a complemented set $A$ (i.e. a couple $A=\left(A_{1}, A_{2}\right)$ of subsets of $\Omega$ such that $\omega_{1} \neq \omega_{2}$ whenever $\omega_{1} \in A_{1}$ and $\omega_{2} \in A_{2}$ ) has indicator function (i.e. the function $1_{A}$ which is 1 on $A_{1}$ and 0 on $A_{2}$ ) belonging to $L^{1}$, then $A$ is called an $\left(L^{1}-\right)$ measurable set (or an $L^{1}$-event). An ( $\left.L^{1}-\right)$ measurable function is a function $X$ on $\Omega$ for which $K \wedge|X|$ belongs to $L^{1}$ for every number $K$. If $X$ is measurable, then for almost every (Lebesgue) real number $a$ the complemented set $(\{\omega: X(\omega) \leqq a\},\{\omega: X(\omega)>a\})$ is measurable. (We will denote this measurable set simply by its first component, or by $(X \leqq a)$, and call the number $P(X \leqq a)=E\left(1_{(X \leqq a)}\right)$ its probability, or measure. This theorem as well as the constructive versions of the standard measure theoretic tools are given in [1] and [2]. In the following, a measurable function $X$ is said to be almost uniformly bounded if, for some sequence $a_{n} \uparrow \infty$, the probabilities $P\left(|X| \leqq a_{n}\right)$ converge to 1 . [Classically, every measurable function is almost uniformly bounded. Constructively, proving this assertion is equivalent to proving that a monotone sequence of integrable functions converging to an integrable function at each point in a measurable set with measure one converges almost uniformly. No proof or counterexample is known.] Suppose $X_{1}, \ldots, X_{n}$ are almost uniformly bounded measurable functions on $\Omega$. Then they induce a probability $E^{\prime}$ on $\left(R^{n}, C\left(R^{n}\right)\right)$, by $E^{\prime}(f)=E\left(f\left(X_{1}, \ldots, X_{n}\right)\right)$ for $f \in C\left(R^{n}\right)$. (Unless otherwise specified, $R^{n}$ is given the Euclidean metric.)

2. Kolmogorov's Theorem. A measurable stochastic process on a probability space $(\Omega, L, E)$ with parameter set $T=[0,1]$ is a measurable function $X$ on $[0,1] \otimes \Omega$. The process $X$ is said to be continuous in probability if

for all $t \in[0,1]$, the function $X(t, \cdot)$ is an almost uniformly bounded random variable on $\Omega$,

and if, when we write $E_{t_{1}, \ldots, t_{n}}(f)$ for $E\left(f\left(X\left(t_{1}, \cdot\right), \ldots, X\left(t_{n}, \cdot\right)\right)\right)$,

for every $f \in C\left(R^{n}\right)$, the function $E_{t_{1}, \ldots, t_{n}}(f)$ in $t_{1}, \ldots, t_{n}$ is continuous on $[0,1]^{n}$. 
For every finite sequence $\sigma=\left(t_{1}, \ldots, t_{n}\right)$ we thus have a probability space $\left(R^{n}, C\left(R^{n}\right), E_{\sigma}\right)$, called the finite joint probability space induced by $X$ at the instants $t_{1}, \ldots, t_{n}$. The family $\left\{E_{\sigma}\right\}$ is consistent, i.e. for every $\sigma=\left(t_{1}, \ldots, t_{n}\right)$ and every map $i:\{1, \ldots, m\} \rightarrow\{1, \ldots, n\}$, we have, for all $f \in C\left(R^{m}\right)$,

$$
\begin{aligned}
\int_{R^{n}} f\left(x_{i(1)}, \ldots, x_{i(m)}\right) d E_{t_{1}, \ldots, t_{n}}\left(x_{1}, \ldots, x_{n}\right) \\
=\int_{R^{m}} f\left(y_{1}, \ldots, y_{m}\right) d E_{t_{i(1)}, \ldots, t_{t(m)}}\left(y_{1}, \ldots, y_{m}\right) .
\end{aligned}
$$

For a family $\left\{\left(R^{n}, C\left(R^{n}\right), E_{\sigma}\right)\right\}$ which satisfies (2.3), the condition (2.2) is equivalent to the seemingly weaker condition

$$
\int_{R^{2}} 1 \wedge\left|x_{1}-x_{2}\right| d E_{r, s}\left(x_{1}, x_{2}\right) \rightarrow 0 \text { as }|r-s| \rightarrow 0 .
$$

Condition (2.4) is in turn equivalent to

for every $\varepsilon>0$, there exists $h>0$ such that $P\left(\left|X_{r}-X_{s}\right| \geqq a\right)<\varepsilon$

whenever $|r-s|<h$ and $a$ is such that $\left(\left|X_{r}-X_{s}\right| \geqq a\right)$ is measurable.

This last condition is usually used as the definition of continuity in probability.

We prove the following constructive version of Kolmogorov's Theorem.

THEOREM 1. Suppose for each sequence $\sigma=\left(t_{1}, \ldots, t_{n}\right)$ of points in $[0,1]$ we are given a probability space $\left(R^{n}, C\left(R^{n}\right), E_{\sigma}\right)$ such that the consistency condition (2.3) and the continuity condition (2.2) are satisfied. Then there exists a measurable process which is continuous in probability and which induces $E_{\sigma}$ as finite joint probability at the sequence $\sigma=\left(t_{1}, \ldots, t_{n}\right)$.

Proof. For the basic set take $\Omega=\bar{R}^{D}$, the set of all functions from the dyadic numbers $D=\left\{k 2^{-n}: 0 \leqq k \leqq 2^{n}\right\}$ to $\bar{R}$, the completion of $R$ under the metric $d_{1}(x, y)=\left|\tan ^{-1} x-\tan ^{-1} y\right|$. Equip $\Omega$ with the compact metric $d\left(\omega, \omega^{\prime}\right)$ $=\sum_{k=1}^{\infty} 2^{-k} d_{1}\left(\omega\left(d_{k}\right), \omega^{\prime}\left(d_{k}\right)\right)$ where $d_{1}, d_{2}, \ldots$ is a fixed enumeration of $D$. Take any continuous function $Y$ on $\Omega$ which depends only on finitely many coordinates, i.e. for which there exists a function $f$ on $\bar{R}^{n}$, and dyadic numbers $t_{1}, \ldots, t_{n}$, such that $Y(\omega)=f\left(\omega\left(t_{1}\right), \ldots, \omega\left(t_{n}\right)\right)$ for every $\omega \in \Omega$. For such a function $f$, the function $f \mid R^{n}$ clearly belongs to $B C\left(R^{n}\right)$, and so $E_{t_{1}, \ldots, t_{n}}(f)$ is defined. With notations as above, write

$$
E(Y)=E_{t_{1}, \ldots, t_{n}}(f)=\int_{R^{n}} f\left(y_{1}, \ldots, \dot{y}_{n}\right) d E_{t_{1}, \ldots, t_{n}}\left(y_{1}, \ldots, y_{n}\right) .
$$

Using the consistency condition (2.3) one easily verifies that $E(Y)$ is well defined. One also sees that $E$ is linear and nonnegative, and takes the constant function 1 to 1 . Now, the continuous functions depending only on finitely many coordinates being dense in $C(\Omega)$ with respect to the supremum norm, the function $E$ 
can be extended to $C(\Omega)$ and we have the probability space $(\Omega, L, E)$ where $L=C(\Omega)$.

For each $r$ in $D$, define $X(r, \omega)=\omega(r)$ if $\omega(r) \in R$. Then $X(r, \cdot)$ is $\left(L^{1-}\right)$ measurable and almost uniformly bounded. Now construct for each $n$ a function $X^{n}$ on $[0,1] \otimes \Omega$ by

$$
X^{n}(t, \omega)=\sum_{k=0}^{2^{n}} g_{k}^{n}(t) X\left(k 2^{n}, \omega\right)
$$

where the function $g_{k}^{n}$ on $[0,1]$ has value 0 at $j 2^{-n}\left(j=0, \ldots, 2^{n}, j \neq k\right)$, has value 1 at $k 2^{-n}$, and is linear on each interval $\left[(j-1) 2^{-n}, j 2^{-n}\right]\left(j=1, \ldots, 2^{n}\right)$. In other words, $X^{n}(\cdot ; \omega)$ is a piecewise linear function on $[0,1]$ which has value $X\left(k 2^{-n}, \omega\right)$ at $k 2^{-n}\left(k=0, \ldots, 2^{n}\right)$. Since the $g_{k}^{n}$ 's are continuous, we see that $X^{n}$ is measurable on $[0,1]$.

Now for every $r, s$ in $D$, we have, by (2.6),

$$
E(1 \wedge|X(r, \cdot)-X(s, \cdot)|)=\int_{R^{2}} 1 \wedge\left|x_{1}-x_{2}\right| d E_{r, s}\left(x_{1}, x_{2}\right) .
$$

Therefore, from the given continuity condition which, as remarked earlier, is equivalent to (2.4), we have

$$
E(1 \wedge|X(r, \cdot)-X(s, \cdot)|) \rightarrow 0 \quad \text { as }|r-s| \rightarrow 0 \quad \text { with } r, s \text { in } D .
$$

Choose a sequence of natural numbers $n_{1}<n_{2}<\cdots$ such that

$$
E(1 \wedge|X(r, \cdot)-X(s, \cdot)|) \leqq 2^{-p} \quad \text { if }|r-s| \leqq 2^{-n_{p}}, r, s \in D .
$$

Suppose $n, m>n_{p}$. Then if $t \in\left[(k-1) 2^{-n}, k 2^{-n}\right] \cap\left[(j-1) 2^{-m}, j 2^{-m}\right]$, then by construction $X^{n}(t, \cdot)=a X\left((k-1) 2^{-n}, \cdot\right)+b X\left(k 2^{-n}, \cdot\right)$ where $a=k-2^{n} t$ and $a+b=1$. Thus

$E\left(1 \wedge\left|\left(X^{n}(t, \cdot)-X\left(k 2^{-n}, \cdot\right)\right)\right|\right)=E\left(1 \wedge a\left|X\left((k-1) 2^{-n}, \cdot\right)-X\left(k 2^{-n},.\right)\right|\right) \leqq 2^{-p}$ by (2.7). Similarly

$$
E\left(1 \wedge\left|X^{m}(t, \cdot)-X\left(j 2^{-m}, \cdot\right)\right|\right) \leqq 2^{-p} .
$$

Combining these two inequalities with $E\left(1 \wedge\left|X\left(k 2^{-n}, \cdot\right)-X\left(j 2^{-m}, \cdot\right)\right|\right) \leqq 2^{-p}$, we have

$$
E\left(1 \wedge\left|X^{m}(t, \cdot)-X^{n}(t, \cdot)\right|\right) \leqq 3 \cdot 2^{-p} .
$$

Since the left-hand side of (2.9) is continuous in $t$, this inequality holds for all $t \in[0,1]$. In particular

$$
E\left(1 \wedge\left|X^{n_{p}}(t, \cdot)-X^{n_{p+1}}(t, \cdot)\right|\right) \leqq 6 \cdot 2^{-p} \text { for } t \in[0,1] .
$$

Consequently $X^{n}$ p converges almost uniformly on $[0,1] \otimes \Omega$, and for each $t$, the sequence $X^{n_{p}}(t, \cdot)$ converges almost uniformly on $\Omega$. Define

$$
X(t, \omega)=\lim _{p \rightarrow \infty} X^{n_{p}}(t, \omega)
$$


whenever this limit exists. Being the almost uniform limit of measurable functions, the function $X$ is measurable on $[0,1] \otimes \Omega$. Since for each $t, X^{n_{p}}(t, \cdot)$ is an almost uniformly bounded measurable function on $\Omega$, so is $X(t, \cdot)$. If we give the set of measurable functions on $\Omega$ the metric $\rho(Y, Z)=E(1 \wedge|Y-Z|)$, then $X$ can be regarded as the uniform limit of the continuous curves $X^{n_{p}}$ in this metric space, hence itself a continuous curve. This implies that the finite joint probabilities $E_{\sigma}^{\prime}$ induced by $X$ satisfy (2.4), and so (2.3). Hence $X$ is continuous in probability.

Finally for each $f \in C\left(R^{n}\right)$, the equality

$$
E\left(f\left(X\left(t_{1}, \cdot\right), \ldots, X\left(t_{n}, \cdot\right)\right)\right)=E_{t_{1}, \ldots, t_{n}}(f)
$$

holds by (2.6) for dyadic numbers $t_{1}, \ldots, t_{n}$. Since both sides are continuous in $t_{1}, \ldots, t_{n}$, they are identical. In other words, $X$ induces $E_{t_{1}, \ldots, t_{n}}$ at $\left(t_{1}, \ldots, t_{n}\right)$.

From the proof we see that $X$ is separable in the following sense. There exist natural numbers $n_{1}<n_{2}<\cdots$ such that for almost every $\omega \in \Omega$, if we let $X^{n}(\cdot, \omega)$ be the function on $[0,1]$ which is linear on $\left[(j-1) 2^{-n}, j 2^{-n}\right]$ and which has value $X\left(k 2^{-n}, \omega\right)$ at $k 2^{-n}$, then $X(t, \omega)$ is defined and equal to $\lim _{p \rightarrow \infty} X^{n} p(t, \omega)$, if the limit exists. This separability would enable us to obtain classically many random variables, e.g. $\sup _{t \in[0,1]} X(t, \cdot)$. Constructively, however, not even $\sup _{d \in D} X(d, \cdot)$ is measurable in general, where $D$ is the set of dyadic numbers, say. [Following is a counterexample. Let $f$ be any fixed bounded measurable function on the real line. Then it is easy to show that the function $X(t, \omega)=f(t-\omega)$ is a measurable process, continuous in probability, on $\Omega=[0,1]$ (with the Lebesgue measure). Now suppose $f=1_{[-a, a]}$ with $a \geqq 0$. Then clearly $\sup _{d \in D} X(d, \cdot)$ equals 1 almost everywhere if $a>0$, and equals 0 almost everywhere if $a=0$. Thus if $\sup _{d \in D} X(d, \cdot)$ were measurable in general, then by computing its integral $E\left(\sup _{d \in D} X(d, \cdot)\right)$ we would be able to decide whether $a=0$, or $a=0 \Rightarrow 0=1$. This is, of course, not possible constructively.] To obtain interesting random variables (which include substitutes for the one just described), we rely heavily on the measurability of $X$.

3. Some stopping times. Let $B$ be a measurable subset of $[0,1] \otimes \Omega$. By Fubini's Theorem, for almost every $\omega \in \Omega$ and for all $s \in[0,1]$, the function $f(s, \omega)=\int_{0}^{s} 1_{\{t:(t, \omega) \in B\}} d t$ is defined. $f$ is a measurable process. In particular, for almost every $\varepsilon>0$, the set $A(\varepsilon)=(f(1, \cdot)<\varepsilon)$ is a measurable subset of $\Omega$. Its measure can be interpreted as the probability that the process spends less than $\varepsilon$ of the time in $B$. [An interesting case is when $B=\{(t, \omega): Y(t, \omega) \in A\}$ where $Y$ is some measurable process continuous in probability, and where $A$ is some subset of $R$ such that $B$ is measurable. Then $f$ intuitively records the time spent by $Y$ in $A$ up to a particular instant. When $A=(a, \infty)$, the measure of $A(\varepsilon)$ gives the probability that the process $Y$ exceeds $a$ for less than $\varepsilon$ of the time. This should be a good substitute for the probability that $Y$ never exceeds $a$, which was shown to be not computable in the example in the last section.] Ignoring a null set, we may 
assume that, for all $\omega \in \Omega$, and for $s \leqq r$ in $[0,1]$,

$$
f(0, \omega)=0 \text { and } 0 \leqq f(r, \omega)-f(s, \omega) \leqq r-s .
$$

For each natural number $n$, define a random variable on $[0,1] \otimes \Omega$ by

$$
\tau^{n}(t, \omega)=\sum_{k=1}^{2^{n}} k 2^{-n} 1_{\left\{(t, \omega): f\left((k-1) 2^{-n}, \omega\right)<t \leqq f\left(k 2^{-n}, \omega\right)\right\}}+1_{\{(t, \omega): f(1, \omega)<t\}} .
$$

In words $\tau^{n}(t, \omega)$ is the smallest number among $k 2^{-n}\left(k=1, \ldots, 2^{n}\right)$ such that $t \leqq f\left(k 2^{-n}, \omega\right)$, if such a number exists. It follows from the monotonicity of $f(\cdot, \omega)$ that, if $n \leqq m, 0 \leqq \tau^{m}(t, \omega)-\tau^{n}(t, \omega) \leqq 2^{-n}$ whenever $\tau^{m}$ and $\tau^{n}$ are defined at $(t, \omega)$. In particular, $\tau^{n}$ converges uniformly on a set of measure one to some random variable $\tau$ on $[0,1] \otimes \Omega$. Suppose $\tau(t, \omega)<1$. From (3.2) we have

$$
f\left(\tau^{n}(t, \omega)-2^{-n}, \omega\right)<t \leqq f\left(\tau^{n}(t, \omega), \omega\right)
$$

for $n$ large enough. Letting $n \rightarrow \infty$ and using the continuity of $f(\cdot, \omega)$, we have

$$
f(\tau(t, \omega), \omega)=t
$$

Moreover, if $s<\tau(t, \omega)$, then for large $n$ we have $s<\tau^{n}(t, \omega)-2^{-n}$ and so $f(s, \omega)$ $\leqq f\left(\tau^{n}(t, \omega)-2^{-n}, \omega\right)<t$. Combining, we see

$$
\tau(t, \omega)=\inf \{s \in[0,1]: s=1 \text { or } f(s, \omega) \geqq t\} .
$$

For this reason $\tau$ is called the first crossing time of $f$. [Classically, (3.4) (with $[0,1]$ replaced by a dense subset) can be used as the definition of $\tau$. But to prove constructively that $\tau$ is measurable, we need to show that it is the limit in some sense of measurable functions.] If $B=\{(t, \omega): Y(t, \omega) \in A\}$ as described before, then $\tau(t, \omega)$ is the first instant when $Y(\cdot, \omega)$ has spent no less than $t$ of the time in $A$. For a small $t, E(\tau(t, \cdot))$ is the constructive substitute for the expected time of hitting $A$ by $Y$. It should be pointed out that $\tau(t, \cdot)$ is a random variable only for almost every $t$.

Now suppose $X$ is a measurable process on $\Omega$, continuous in probability, and separable in the sense described in the last section. Let $f$ be a function on $[0,1] \otimes \Omega$ satisfying (3.1), with the first crossing time $\tau$ associated as above.

THEOREM 2. Under the above assumptions, for almost every $t \in[0,1]$, the function $X(\tau(t, \cdot), \cdot)$ is measurable on $\Omega$.

[Classically this theorem would hold for every $t$. However, the random variables $X(\tau(t, \cdot), \cdot)$ (as well as the others) which we obtain are intrinsic of the process, i.e. the same (up to equivalence) for two processes if they induce identical finite joint probabilities. This is certainly not the case classically: $E(X(\tau(t, \cdot), \cdot))$ can be quite arbitrary.] 
Proof. For any real number $a \in[0,1)$ such that $(\tau \geqq a)=\{(t, \omega): \tau(t, \omega) \geqq a\}$ is measurable, we have

$$
\begin{aligned}
& \iint_{(\tau \leqq a)} d E(\omega) d t 1 \wedge\left|X^{n_{p}}(\tau(t, \omega), \omega)-X^{n_{q}}(\tau(t, \omega), \omega)\right| \\
& =\int d E(\omega) \int_{\{t: \tau(t, \omega) \leqq a\}} 1 \wedge\left|X^{n_{p}}(\tau(t, \omega), \omega)-X^{n_{q}}(\tau(t, \omega), \omega)\right| \\
& =\int d E(\omega) \int_{0}^{a} d f(s, \omega) 1 \wedge\left|X^{n_{p}}(s, \omega)-X^{n_{q}}(s, \omega)\right| \\
& \leqq \int d E(\omega) \int_{0}^{1} d s 1 \wedge\left|X^{n_{p}}(s, \omega)-X^{n_{q}}(s, \omega)\right| \text {. }
\end{aligned}
$$

The second equality follows formally from the change of variables $s=\tau(t, \omega)$, $t=f(s, \omega)$ (see (3.3)) whose justification is easy and omitted. The inequality follows from (3.1).

Now let $\varepsilon>0$ be arbitrary. In view of the continuity (in probability) of $X$, we can make the right-hand side of (3.5) smaller than $\varepsilon$ by making $p$ and $q$ large enough. Let such a pair of integers $p$ and $q$ be fixed. Suppose $a \in\left(1-2^{-n_{p}}, 1\right) \cap\left(1-2^{-n_{q}}, 1\right)$ is such that $(\tau \leqq a)$ is measurable. Then for $(t, \omega)$ in $(\tau>a)$ we have, by the definition of $X^{n}$,

$$
\begin{aligned}
& 1 \wedge\left|X^{n_{p}}(\tau(t, \omega), \omega)-X^{n_{q}}(\tau(t, \omega), \omega)\right| \\
& =1 \wedge \mid(1-\tau(t, \omega)) 2^{n_{p}} X\left(1-2^{-n_{p}}, \omega\right)+\left(\tau(t, \omega)-1+2^{-n_{p}}\right) 2^{n_{p}} X(1, \omega) \\
& -(1-\tau(t, \omega)) 2^{n_{a}} X\left(1-2^{-n_{q}}, \omega\right)-\left(\tau(t, \omega)-1+2^{-n_{a}}\right) 2^{n_{a}} X(1, \omega) \\
& \leqq 1 \wedge(1-\tau(t, \omega))\left[2^{n_{p}}\left|X\left(1-2^{-n_{p}}, \omega\right)-X(1, \omega)\right|\right. \\
& \left.+2^{n_{q}}\left|X\left(1-2^{-n_{q}}, \omega\right)-X(1, \omega)\right|\right] \\
& \leqq 1 \wedge(1-a)\left[2^{n_{p}}\left|X\left(1-2^{-n_{p}}, \omega\right)-X(1, \omega)\right|+2^{n_{q}}\left|X\left(1-2^{-n_{q}}, \omega\right)-X(1, \omega)\right|\right] .
\end{aligned}
$$

Since the random variables $X\left(1-2^{-n_{p}}, \cdot\right), X\left(1-2^{-n_{q}}, \cdot\right)$, and $X(1, \cdot)$ are almost uniformly bounded, the last expression in the above inequalities converges almost uniformly to 0 as $a$ approaches 1 . Hence we can choose the number $a$ so near to 1 that the first integral in (3.5), with $(\tau \leqq a)$ replaced by $(\tau>a)$, is bounded by $\varepsilon$. Combining, we see that

$$
\iint d E(\omega) d t 1 \wedge\left|X^{n_{p}}(\tau(t, \omega), \omega)-X^{n_{q}}(\tau(t, \omega), \omega)\right| \leqq 2 \varepsilon .
$$

Passing to a subsequence, we may assume that $X^{n} p(\tau(t, \omega), \omega)$ converges almost uniformly on $[0,1] \otimes \Omega$. By the definition of separability, the limit must be equal, almost everywhere on $[0,1] \otimes \Omega$, to $X(\tau(t, \omega), \omega)$. Hence the latter is a random variable on $[0,1] \otimes \Omega$. In particular, for almost every $t$ in $[0,1], X(\tau(t, \cdot), \cdot)$ is a random variable on $\Omega$. This finishes the proof.

Let $f$ and $X$ be measurable processes on $(\Omega, L, E)$, continuous in probability, with $f$ satisfying (3.1). Suppose $\left\{L_{t}: t \in[0,1]\right\}$ is a family of linear subspaces of $L^{1}$, closed under $\wedge$ and containing constants, such that $L_{r} \subset L_{s}$ if $r \leqq s$. Then 
$\left(\Omega, L_{t}, E\right)$ is a probability space for every $t$. Suppose further that $f$ and $X$ are adapted to the family $L_{t}$ in the sense that $f(t, \cdot)$ and $X(t, \cdot)$ belong to $L_{t}^{1}$ for every $t$. Let $\tau$ denote the first crossing time for $f$. Then we can prove that for almost every $s \in[0,1]$, the random variable $\tau(s, \cdot)$ is a stopping time for $\left\{L_{t}\right\}$, in the sense that if $g$ is a continuous function on $[0,1]$ supported by $[0, t]$, then $g(\tau(s, \cdot)) \in L_{t}^{1}$. It follows that for almost every $t \in[0,1]$, the set $(\tau(s, \cdot) \leqq t)$ is $L_{t}^{1}$-measurable. If we write

$$
L_{\tau(s, \cdot)}=\left\{Y \in L^{1}: Y \cdot g(\tau(s, \cdot)) \in L_{t}^{1} \text { if } g \in C([0,1]) \text { is supported by }[0, t]\right\},
$$

then $\left(\Omega, L_{\tau(s, .)}, E\right)$ is a probability space. It can be proved that for almost every $s$, the random variable $X(\tau(s, \cdot), \cdot)$ is actually $L_{\tau(s, \cdot)}$-measurable.

It has recently been pointed out to the author that a similar approach was used by Nelson [3, Theorem 1], in constructing a process from given finite joint probabilities. Following [3], one would take the basic set $\Omega$ to be $\bar{R}^{[0,1]}$ with the compact product topology. Constructively, however, even the product $\bar{R}^{\{a, b\}}=\{f: f$ is a function from $\{a, b\}$ to $\bar{R}\}$ fails to be compact under the product metric $d\left(f, f^{\prime}\right)$ $=\left|\tan ^{-1} f(a)-\tan ^{-1} f^{\prime}(a)\right|+\left|\tan ^{-1} f(b)-\tan ^{-1} f^{\prime}(b)\right|$, unless we know either $a=b$ or $a \neq b$. This difficulty is avoided in the present paper by using the product $\bar{R}^{D}$ where $D$ is as nice a set as the dyadic numbers-we can certainly tell whether two dyadic numbers are equal. In view of the continuity (2.2), we know the whole process probabilistically if we know it at the dense set of dyadic numbers, and so $\bar{R}^{D}$ is obviously an appropriate choice. No continuity in probability need be assumed in [3]. Actually, however, the continuity condition (2.2) is hardly a restriction if we assume that $X(t, \cdot)$ is a random variable for all $t$. For then

$$
E\left(f\left(X\left(t_{1}, \cdot\right)\right), \ldots, f\left(X\left(t_{n}, \cdot\right)\right)\right)
$$

is a function defined everywhere on $[0,1]^{n}$, and we have not seen such a function except the continuous one.

This paper is an outgrowth of part of a doctoral dissertation written at the University of California, San Diego, under the guidance of Professor Errett Bishop, whom the author wishes to thank for his advice and encouragement.

\section{REFERENCES}

1. E. Bishop, Foundations of constructive analysis, McGraw-Hill, New York, 1967. MR 36 \#4930.

2. E. Bishop and H. Cheng, Constructive measure theory (to appear).

3. E. Nelson, Regular probability measures on function space, Ann. of Math. (2) 69 (1959), 630-643. MR 21 \#4479.

4. J. Neveu, Bases mathématiques du calcul des probabilités, Masson, Paris, 1964; English transl., Holden-Day, San Francisco, Calif., 1965. MR 33 \#6659; MR 33 \#6660.

Department of Mathematics, University of Washington, Seattle, Washington 98105 